\title{
Polynuclear Metallacarborane-Hydrocarbon Assemblies: Metallacarborane
}

\author{
Dendrimers \\ Haijun Yao and Russell N. Grimes* \\ Department of Chemistry, University of Virginia, Charlottesville, VA 22901 \\ Maddalena Corsini and Piero Zanello \\ Dipartimento di Chimica, Università di Siena, Via Aldo Moro, 53100 Siena, Italy
}

\section{Supporting Information}

Instrumentation. ${ }^{1} \mathrm{H}(500 \mathrm{MHz}$ [where noted], $300 \mathrm{MHz}),{ }^{13} \mathrm{C}(125.8 \mathrm{MHz})$, and ${ }^{11} \mathrm{~B}(96.4 \mathrm{MHz}) \mathrm{NMR}$ spectra were recorded on GN-300/44 and Varian Unity/Inova 500/51 and 300/51 instruments. ${ }^{1} \mathrm{H}$ and ${ }^{13} \mathrm{C}$ shifts are referenced to residual ${ }^{1} \mathrm{H}$ and ${ }^{13} \mathrm{C}$ signals in the deuterated solvent. ${ }^{11} \mathrm{~B}$ NMR resonances are referenced to the external standard $\mathrm{BF}_{3} \bullet \mathrm{OEt}_{2}$. Unit resolution mass spectra were acquired on a Finnigan (Model LCQ Classic) quadruple ion trap mass spectrometer using an atmospheric pressure chemical ionization interface. Matrix assisted laser desorption lonization-time of flight mass spectra were acquired at the W. M. Keck Biomedical Mass Spectrometry Laboratory of the University of Virginia on an ABI Voyager DE-Pro instrument, using sinapinic acid as a matrix. Infrared spectra were obtained on a Nicolet Impact400 spectrophotometer. Ultraviolet-visible spectra were recorded on a Cary 5E UV-VIS-NIR Spectrophotometer.

Materials and Procedures. All reactions were carried out in oven glassware under a dinitrogen atmosphere using conventional glove box or Schlenk techniques, and the products were worked up in air. All commercial reagents were used as received without further purification. THF, diethyl ether were distilled from sodium benzophenone ketyl prior to use. Triethylamine was distilled from $\mathrm{CaH}_{2}$ under an inert atmosphere. Anhydrous dichloromethane was distilled from $\mathrm{P}_{2} \mathrm{O}_{5}$ under $\mathrm{N}_{2}$. $\mathrm{CpCo}\left(2,3-\mathrm{Et}_{2} \mathrm{C}_{2} \mathrm{~B}_{4} \mathrm{H}_{3}\right)$ (1) was prepared by the published procedure. ${ }^{1}$

Materials and apparatus employed in the electrochemical studies have been described elsewhere. ${ }^{2}$ Under the experimental conditions employed in this work, the one-electron oxidation of ferrocene at the gold electrode ( $3 \mathrm{~mm}$ diameter disk) occurs at $\mathrm{E}^{\circ \prime}=+0.51 \mathrm{~V}$, vs. SCE, representatively with $\Delta \mathrm{E}_{\mathrm{p}}=112 \mathrm{mV}$ at $0.05 \mathrm{Vs}^{-1}$

$\left[\eta^{5}-\mathrm{C}_{5} \mathrm{H}_{4} \mathrm{C}(\mathrm{O}) \mathrm{OH}\right] \mathrm{Co}\left(2,3-\mathrm{Et}_{2} \mathrm{C}_{2} \mathrm{~B}_{4} \mathrm{H}_{4}\right)(2)$. To a solution of 1 (760 mg, $\left.3 \mathrm{mmol}\right)$ in $20 \mathrm{~mL}$ of THF was added $3 \mathrm{mmol}$ of $n$-butyllithium $\left(1.88 \mathrm{~mL}\right.$ of a $1.6 \mathrm{M}$ solution in hexane) at $-78{ }^{\circ} \mathrm{C}$. The reaction mixture was stirred at $-78{ }^{\circ} \mathrm{C}$ for $1 \mathrm{~h}$ and transferred to a mixture of finely crushed dry ice in $40 \mathrm{~mL}$ anhydrous ethyl ether with rapid stirring. The mixture was stirred at room temperature overnight under nitrogen, opened to air, $1 \mathrm{~mL}$ of concentrated hydrochloric acid was added, and the mixture was stirred for $10 \mathrm{~min}$. The organic 
portion was washed with $50 \mathrm{~mL}$ of $\mathrm{H}_{2} \mathrm{O}$ three times and dried over anhydrous $\mathrm{Na}_{2} \mathrm{SO}_{4}$. After removal of solvent, the residue was washed with hexane to give $410 \mathrm{mg}$ of product as an orange red solid $(46 \%, 58 \%$ based on starting material consumed). Removal the solvent from the hexane wash afforded $158 \mathrm{mg}$ of recovered starting material. ${ }^{1} \mathrm{H}$ NMR $\left(500 \mathrm{MHz}_{\mathrm{CDCl}}\right): \delta=1.31$ (t, $6 \mathrm{H}, \mathrm{J}=7.5 \mathrm{~Hz}$, ethyl $\left.\mathrm{CH}_{3}\right), 2.43,2.69$ (sextet, $4 \mathrm{H}, J=7 \mathrm{~Hz}$, ethyl $\left.\mathrm{CH}_{2}\right), 5.05\left(\mathrm{t}, 2 \mathrm{H}, J=2.3 \mathrm{~Hz}, \mathrm{C}_{5} \mathrm{H}_{4}\right), 5.31\left(\mathrm{t}, 2 \mathrm{H}, J=2.3 \mathrm{~Hz}, \mathrm{C}_{5} \mathrm{H}_{4}\right), 11.38(1 \mathrm{H}$, $\mathrm{COOH}$ ). ${ }^{13} \mathrm{C}\left\{{ }^{1} \mathrm{H}\right\}$ NMR $\left(\mathrm{CDCl}_{3}\right): \delta=14.7$ (ethyl $\left.\mathrm{CH}_{3}\right), 24.0$ (ethyl $\left.\mathrm{CH}_{2}\right), 82.2\left(\mathrm{C}_{5} \mathrm{H}_{4}\right), 84.2(\mathrm{Cp}), 98.8\left(\mathrm{C}_{2} \mathrm{~B}_{4}\right)$, $171.4(\mathrm{C}=\mathrm{O}) .{ }^{11} \mathrm{~B}$ NMR $\left(\mathrm{CDCl}_{3}\right): \delta=3.7(\mathrm{BH}, 2 \mathrm{~B}$, unresolved ), $11.7(\mathrm{BH}, 2 \mathrm{~B}$, unresolved). $\mathrm{IR}(\mathrm{KBr}$ pellet, $\left.\mathrm{cm}^{-1}\right): v=3125.4(w), 3108.4(w), 2969.0(\mathrm{~m}), 2934.9(w), 2872.9(w), 2550.6(\mathrm{~s}, \mathrm{~B}-\mathrm{H}), 1697.3(\mathrm{vs}, \mathrm{C}=\mathrm{O})$, 1485.0 (s), $1409.1(\mathrm{~m}), 1290.5(\mathrm{~s}), 1173.7(\mathrm{~m}), 1060.4(\mathrm{w}), 1034.5(\mathrm{w}), 912.0(\mathrm{w}), 852.4(\mathrm{~m}), 786.6(\mathrm{w})$, $743.8(\mathrm{w}), 594.9(\mathrm{w}), 458.5(\mathrm{~m}) . \mathrm{Cl}^{+}$-Mass: $\mathrm{m} / \mathrm{z}(\%)=298.6\left(\left[\mathrm{M}^{+}\right], 100\right)$.

$\left[\eta^{5}-\mathrm{C}_{5} \mathrm{H}_{4} \mathrm{C}(\mathrm{O}) \mathrm{Cl}\right] \mathrm{Co}\left(2,3-\mathrm{Et}_{2} \mathrm{C}_{2} \mathrm{~B}_{4} \mathrm{H}_{4}\right)$ (3). To a solution of $2(308 \mathrm{mg}, 1.04 \mathrm{mmol})$ in $15 \mathrm{~mL}$ of anhydrous benzene in a dry box, $216 \mathrm{mg}$ of $\mathrm{PCl}_{5}(1.04 \mathrm{mmol})$ was added in small portions over a $3 \mathrm{~h}$ period. After $1 \mathrm{~h}$ of stirring, the reaction mixture was washed with dilute aqueous $\mathrm{NaOH}$ twice and with water six times, and the residue was dried over anhydrous $\mathrm{Na}_{2} \mathrm{SO}_{4}$. The solvent was removed, the residue was extracted with hexane, and the hexane removed to give $306 \mathrm{mg}$ of product as a red oil $(94 \%)$. ${ }^{1} \mathrm{H}$ $\operatorname{NMR}\left(\mathrm{CDCl}_{3}\right): \delta=1.30\left(\mathrm{t}, 6 \mathrm{H}, \mathrm{J}=7.5 \mathrm{~Hz}\right.$, ethyl $\left.\mathrm{CH}_{3}\right), 2.45,2.70$ (sextet, $4 \mathrm{H}, J=7.5 \mathrm{~Hz}$, ethyl $\left.\mathrm{CH}_{2}\right), 5.14(\mathrm{t}$, $\left.2 \mathrm{H}, J=2.1 \mathrm{~Hz}, \mathrm{C}_{5} \mathrm{H}_{4}\right), 5.34\left(\mathrm{t}, 2 \mathrm{H}, J=2.1 \mathrm{~Hz}, \mathrm{C}_{5} \mathrm{H}_{4}\right) \cdot{ }^{13} \mathrm{C}\left\{{ }^{1} \mathrm{H}\right\} \mathrm{NMR}\left(\mathrm{CDCl}_{3}\right): \delta=14.6$ (ethyl $\mathrm{CH}_{3}$ ), 23.9 (ethyl $\left.\mathrm{CH}_{2}\right), 82.8\left(\mathrm{C}_{5} \mathrm{H}_{4}\right), 83.5\left(\mathrm{C}_{5} \mathrm{H}_{4}\right), 99.2\left(\mathrm{C}_{2} \mathrm{~B}_{4}\right), 164.5(\mathrm{C}=\mathrm{O}) .{ }^{11} \mathrm{~B} \mathrm{NMR}\left(\mathrm{CDCl}_{3}\right): \delta=3.8\left(\mathrm{BH}, 2 \mathrm{~B}, J_{\mathrm{BH}}=146 \mathrm{~Hz}\right)$, $12.3\left(\mathrm{BH}, 2 \mathrm{~B}, J_{\mathrm{BH}}=134 \mathrm{~Hz}\right)$. IR $\left(\mathrm{KBr}\right.$ pellet, $\left.\mathrm{cm}^{-1}\right): v=3117.8(\mathrm{w}), 2970.5(\mathrm{~m}), 2933.9(\mathrm{w}), 2874.7(\mathrm{w})$, 2563.5 (s, B-H), 1667.2 (vs, C=O), $1731.8(\mathrm{~m}), 1448.2(\mathrm{~m}), 1403.8(\mathrm{w}), 1368.7(\mathrm{~m}), 1245.9(\mathrm{~s}), 1047.1(\mathrm{~m})$, $1034.7(w), 942.0(\mathrm{~m}), 866.6(\mathrm{w}), 842.3(\mathrm{~m}), 792.8(\mathrm{~s}), 710.1(\mathrm{w}), 447.0(\mathrm{w}), 432.0(\mathrm{w}) . \mathrm{Cl}^{+}-\mathrm{Mass}^{\mathrm{m}} \mathrm{m} / \mathrm{z}(\%)=$ $281.1\left(\left[\mathrm{M}^{+}-\mathrm{Cl}\right], 100\right)$.

(2,3-Et $\left.\mathrm{C}_{2} \mathrm{~B}_{3} \mathrm{H}_{5}\right) \mathrm{CoC}_{5} \mathrm{H}_{4} \mathrm{COCl}$ (4). Compound 3 (240 mg, $0.76 \mathrm{mmol}$ ) was dissolved in $4 \mathrm{~mL}$ of ethyl acetate and $1 \mathrm{~mL}$ of water was added. The mixture was stirred vigorously for $10 \mathrm{~h}$. The water was separated, and the ethyl acetate solution was washed with water three times and dried over anhydrous $\mathrm{Na}_{2} \mathrm{SO}_{4}$. The solvent was removed, the residue was taken up in hexane, eluted through $2 \mathrm{~cm}$ of silica gel, and washed with $50 \%$ of hexane/dichloromethane. Removal of the solvents gave $217 \mathrm{mg}$ of product as a red oil (93 \%). ${ }^{1} \mathrm{H}$ NMR $\left(\mathrm{CDCl}_{3}\right): \delta=-5.81$ (s, 2H, B-H-B), 1.15 (t, $6 \mathrm{H}, \mathrm{J}=7.5 \mathrm{~Hz}$, ethyl $\left.\mathrm{CH}_{3}\right), 2.09,2.18$ (sextet, $4 \mathrm{H}, J=7.5 \mathrm{~Hz}$, ethyl $\mathrm{CH}_{2}$ ), $5.10\left(\mathrm{t}, 2 \mathrm{H}, J=2.4 \mathrm{~Hz}, \mathrm{C}_{5} \mathrm{H}_{4}\right), 5.34\left(\mathrm{t}, 2 \mathrm{H}, J=2.4 \mathrm{~Hz}, \mathrm{C}_{5} \mathrm{H}_{4}\right) .{ }^{13} \mathrm{C}\left\{{ }^{1} \mathrm{H}\right\}$ $\operatorname{NMR}\left(\mathrm{CDCl}_{3}\right): \delta=16.0$ (ethyl $\left.\mathrm{CH}_{3}\right), 25.3$ (ethyl $\left.\mathrm{CH}_{2}\right), 85.3\left(\mathrm{C}_{5} \mathrm{H}_{4}\right), 86.9\left(\mathrm{C}_{5} \mathrm{H}_{4}\right), 91.6\left(\mathrm{C}_{2} \mathrm{~B}_{4}\right), 164.9(\mathrm{C}=\mathrm{O})$. ${ }^{11} \mathrm{~B}$ NMR $\left(\mathrm{CDCl}_{3}\right): \delta=4.0\left(\mathrm{BH}, 3 \mathrm{~B}, \mathrm{~J}_{\mathrm{BH}}=120 \mathrm{~Hz}\right)$,). IR $\left(\mathrm{KBr}\right.$ pellet, $\left.\mathrm{cm}^{-1}\right): v=3119.0(\mathrm{w}), 2964.5(\mathrm{~m}), 2930.4$ (w), 2871.3 (w), 2546.6 (s, B-H), 2366.2 (w), 1872.9 (w, B-H-B), 1666.8 (vs, C=O), 1731.7 (m), 1574.2 (w), $1450.0(\mathrm{~m}), 1403.1(\mathrm{w}), 1370.1(\mathrm{~m}), 1248.8(\mathrm{~s}), 1047.1(\mathrm{~m}), 1012.9(\mathrm{w}), 937.3(\mathrm{~m}), 839.5(\mathrm{w}), 798.1(\mathrm{~s})$, $698.8(w), 539.5(w), 443.8(w) . \mathrm{Cl}^{+}-$Mass: $\mathrm{m} / \mathrm{z}(\%)=270.8\left(\left[\mathrm{M}^{+}-\mathrm{Cl}\right], 100\right)$. 
DAB-dend-[NHCO- $\left.\mathrm{C}_{5} \mathrm{H}_{4} \mathrm{Co}\left(2,3-\mathrm{Et}_{2} \mathrm{C}_{2} \mathrm{~B}_{3} \mathrm{H}_{5}\right)\right]_{16}$ (5). A mixture of DAB-Am-16 (30 mg, $\left.0.018 \mathrm{mmol}\right)$ and triethylamine (30 mg, $0.3 \mathrm{mmol}$ ) in $1 \mathrm{~mL}$ of $\mathrm{CH}_{2} \mathrm{Cl}_{2}$ was added dropwise to a stirred solution of 4 (92 $\mathrm{mg}, 0.3 \mathrm{mmol}$ ) in $2 \mathrm{~mL}$ of $\mathrm{CH}_{2} \mathrm{Cl}_{2}$ under nitrogen at room temperature. The reaction mixture was stirred 1 day, after which the solution was washed with saturated $\mathrm{NaHCO}_{3}$ aqueous solution, brine, and water, and dried over anhydrous $\mathrm{MgSO}_{4}$. After removal of the solvent the product was purified by repeated precipitations from $\mathrm{CH}_{2} \mathrm{Cl}_{2} /$ hexane to afford $82 \mathrm{mg}$ of product as an orange-yellow air- stable solid (76\%). ${ }^{1} \mathrm{H}$ NMR $\left(500 \mathrm{MHz}, \mathrm{CDCl}_{3}\right): \delta=-5.96(\mathrm{~s}, 32 \mathrm{H}, \mathrm{B}-\mathrm{H}-\mathrm{B}), 1.13\left(\mathrm{t}, 96 \mathrm{H}, \mathrm{J}=7.5 \mathrm{~Hz}\right.$, ethyl $\left.\mathrm{CH}_{3}\right), 1.34(\mathrm{br}, 4 \mathrm{H}$, $\mathrm{NCH}_{2} \mathrm{CH}_{2} \mathrm{CH}_{2} \mathrm{CH}_{2} \mathrm{~N}$ ), 1.55, 1.75 (br, $56 \mathrm{H}, \mathrm{CH}_{2} \mathrm{CH}_{2} \mathrm{CH}_{2}$ ), 2.10, 2.18 (sextet, $64 \mathrm{H}, J=7 \mathrm{~Hz}$, ethyl $\mathrm{CH}_{2}$ ), 2.39, 2.48 (br, 84H, $\mathrm{CH}_{2} \mathrm{NCH}_{2}$ ), 3.45 (br, 32H, $\left.\mathrm{NHCH}_{2}\right), 4.88\left(\mathrm{t}, 32 \mathrm{H}, \mathrm{C}_{5} \mathrm{H}_{4}\right), 5.41\left(\mathrm{t}, 32 \mathrm{H}, \mathrm{C}_{5} \mathrm{H}_{4}\right), 7.67(\mathrm{t}, 16 \mathrm{H}$, $\mathrm{NH}) .{ }^{13} \mathrm{C}\left\{{ }^{1} \mathrm{H}\right\} \operatorname{NMR}\left(\mathrm{CDCl}_{3}\right): \delta=16.2$ (ethyl $\left.\mathrm{CH}_{3}\right), 25.4$ (ethyl $\left.\mathrm{CH}_{2}\right), 27.1\left(\mathrm{CH}_{2} \mathrm{CH}_{2} \mathrm{CH}_{2}\right), 38.5\left(\mathrm{NHCH}_{2}\right), 51.8$, $52.3\left(\mathrm{CH}_{2} \mathrm{NCH}_{2}\right), 82.2\left(\mathrm{C}_{5} \mathrm{H}_{4}\right), 84.1\left(\mathrm{C}_{5} \mathrm{H}_{4}\right), 92.6(\mathrm{Cp}), 114.2\left(\mathrm{C}_{2} \mathrm{~B}_{4}\right), 164.8(\mathrm{C}=\mathrm{O}) .{ }^{11} \mathrm{~B} \mathrm{NMR}\left(\mathrm{CDCl}_{3}\right): \delta=4.0$ (BH, 3B unresolved). IR (KBr pellet, $\left.\mathrm{cm}^{-1}\right): v=3326.6(\mathrm{~s}, \mathrm{~N}-\mathrm{H}), 3092.3(\mathrm{w}), 2959.3(\mathrm{~s}), 2868.9(\mathrm{~m}), 2810.3$ (m), 2540.1 (s, B-H), 2361.5 (w), 1869.2 (m, B-H-B), 1689.3 (vs, C=O), 1549.6 (vs), 1455.4 (m), 1376.1 (m), 1301.6 (m), 1186.6 (w), 1057.1 (w), 930.6 (w), 837.7 (w), 779.6 (s), 740.5 (w). UV-vis $\left(\mathrm{CH}_{2} \mathrm{Cl}_{2}, \mathrm{~nm}\right.$ (\%)): 273 (100), 320 (19), 369 (6), 587 (2) $\varepsilon_{\max }=217,390 \mathrm{~cm}^{-1} \mathrm{M}^{-1} . \mathrm{MS}(\mathrm{MALDI}-\mathrm{TOF}) \mathrm{m} / \mathrm{z}(\%)=5998\left(\left[\mathrm{M}^{+}-\right.\right.$ 3], 100).

DAB-dend-[NHCO- $\left.\mathrm{C}_{5} \mathrm{H}_{4} \mathrm{Co}\left(2,3-\mathrm{Et}_{2} \mathrm{C}_{2} \mathrm{~B}_{3} \mathrm{H}_{5}\right)\right]_{32}$ (6). A mixture of DAB-Am-32 (72 mg, $0.02 \mathrm{mmol}$ ) and triethylamine $(71 \mathrm{mg}, 0.7 \mathrm{mmol})$ in $2 \mathrm{~mL}$ of $\mathrm{CH}_{2} \mathrm{Cl}_{2}$ was added dropwise to a stirred solution of 4 (200 $\mathrm{mg}, 0.65 \mathrm{mmol}$ ) in $4 \mathrm{~mL}$ of $\mathrm{CH}_{2} \mathrm{Cl}_{2}$ under nitrogen at room temperature. The reaction mixture was worked up as in the case of 5 to afford $192 \mathrm{mg}$ of product as an orange-yellow air stable solid (79\%). ${ }^{1} \mathrm{H}$ NMR (500 $\left.\mathrm{MHz}, \mathrm{CDCl}_{3}\right): \delta=-5.96(\mathrm{~s}, 64 \mathrm{H}, \mathrm{B}-\mathrm{H}-\mathrm{B}), 1.13\left(\mathrm{t}, 192 \mathrm{H}, \mathrm{J}=7.5 \mathrm{~Hz}\right.$, ethyl $\left.\mathrm{CH}_{3}\right), 1.27$ (br, $4 \mathrm{H}$, $\mathrm{NCH}_{2} \mathrm{CH}_{2} \mathrm{CH}_{2} \mathrm{CH}_{2} \mathrm{~N}$ ), 1.61, 1.78 (br, $60 \mathrm{H}, \mathrm{CH}_{2} \mathrm{CH}_{2} \mathrm{CH}_{2}$ ), 2.10, 2.17 (sextet, $128 \mathrm{H}, \mathrm{J}=7 \mathrm{~Hz}$, ethyl $\mathrm{CH}_{2}$ ), 2.45, $2.52\left(\mathrm{br}, 180 \mathrm{H}, \mathrm{CH}_{2} \mathrm{NCH}_{2}\right), 3.43\left(\mathrm{br}, 64 \mathrm{H}, \mathrm{NHCH}_{2}\right), 4.87\left(\mathrm{t}, 64 \mathrm{H}, \mathrm{C}_{5} \mathrm{H}_{4}\right), 5.45\left(\mathrm{t}, 64 \mathrm{H}, \mathrm{C}_{5} \mathrm{H}_{4}\right), 7.86(\mathrm{~s}$, $32 \mathrm{H}, \mathrm{NH}) .{ }^{13} \mathrm{C}\left\{{ }^{1} \mathrm{H}\right\} \mathrm{NMR}\left(\mathrm{CDCl}_{3}\right): \delta=16.3$ (ethyl $\left.\mathrm{CH}_{3}\right), 25.4$ (ethyl $\left.\mathrm{CH}_{2}\right), 26.9\left(\mathrm{CH}_{2} \mathrm{CH}_{2} \mathrm{CH}_{2}\right), 38.3\left(\mathrm{NHCH}_{2}\right)$, 51.6, $52.3\left(\mathrm{CH}_{2} \mathrm{NCH}_{2}\right), 82.2\left(\mathrm{C}_{5} \mathrm{H}_{4}\right), 84.2\left(\mathrm{C}_{5} \mathrm{H}_{4}\right), 92.4(\mathrm{Cp}), 114.1\left(\mathrm{C}_{2} \mathrm{~B}_{4}\right), 164.9(\mathrm{C}=\mathrm{O}) .{ }^{11} \mathrm{~B} \mathrm{NMR}\left(\mathrm{CDCl}_{3}\right): \delta$ $=3.2\left(\mathrm{BH}, 3 \mathrm{~B}\right.$ unresolved). IR (KBr pellet, $\left.\mathrm{cm}^{-1}\right): v=3329.6(\mathrm{~s}, \mathrm{~N}-\mathrm{H}), 3092.0(\mathrm{w}), 2959.6(\mathrm{~s}), 2932.2(\mathrm{~s})$, 2869.2 (m), 2810.7 (w), 2539.3 (s, B-H), 2363.3 (w), 1869.3 (m, B-H-B), 1638.8 (vs, C=O), 1549.7 (vs), $1457.7(\mathrm{~m}), 1378.2(\mathrm{~m}), 1302.0(\mathrm{~m}), 1185.8(\mathrm{w}), 1060.3(\mathrm{w}), 931.3(\mathrm{w}), 837.7(\mathrm{w}), 779.5(\mathrm{~m}), 468.3(\mathrm{w})$. UV-vis $\left(\mathrm{CH}_{2} \mathrm{Cl}_{2}, \mathrm{~nm}(\%)\right): 273$ (100), 321 (18), 379 (6), 589 (2) $\varepsilon_{\max }=474,651 \mathrm{~cm}^{-1} \mathrm{M}^{-1}$. MS (MALDI-TOF) $\mathrm{m} / \mathrm{z}(\%)=12139\left(\left[\mathrm{M}^{+}-3\right], 100\right)$. 

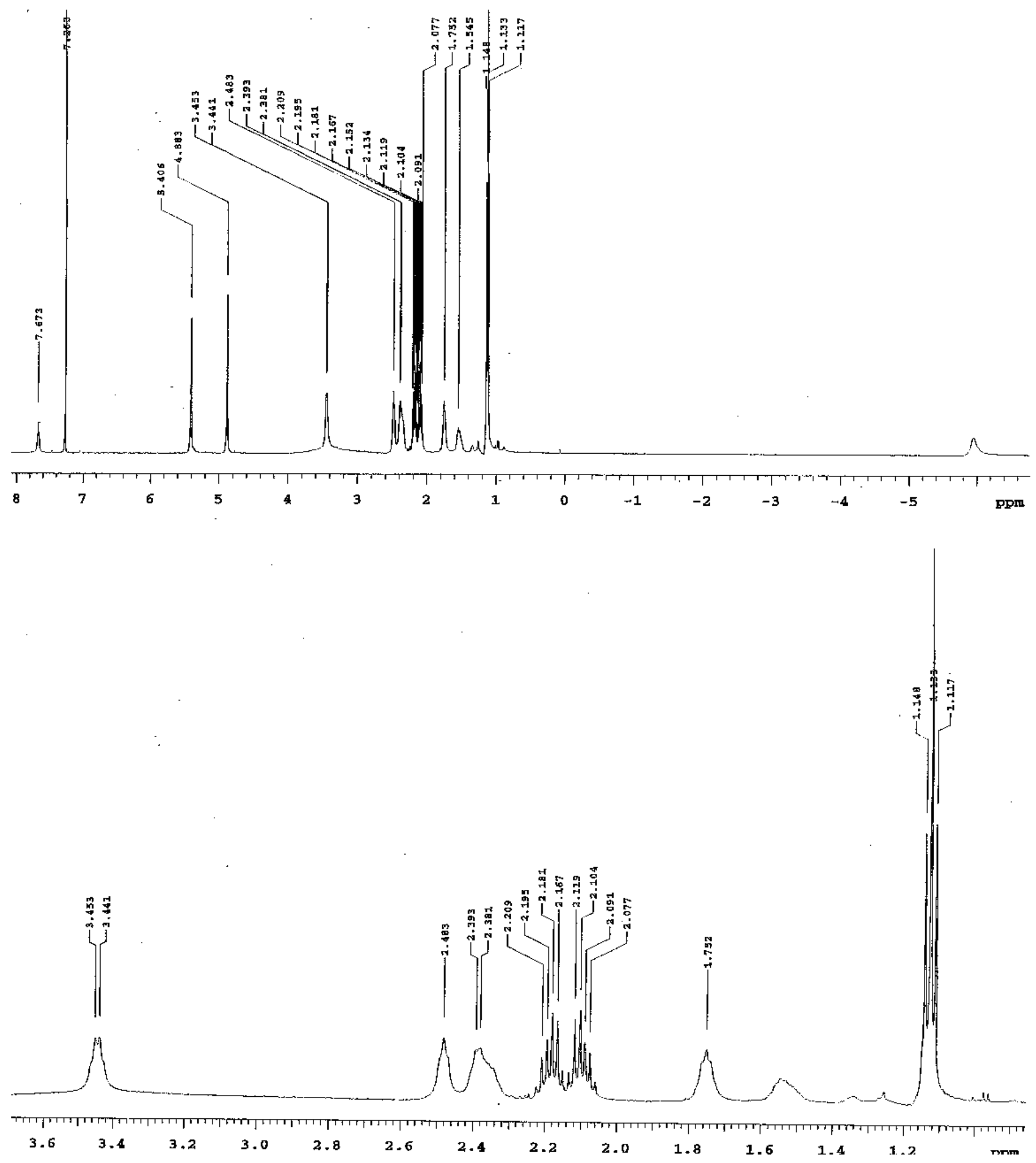

Figure 1 (a). $500 \mathrm{MHz}{ }^{1} \mathrm{H}$ NMR spectra of dend-[NHC(O)- $\left.\mathrm{C}_{5} \mathrm{H}_{4} \mathrm{Co}\left(2,3-\mathrm{Et}_{2} \mathrm{C}_{2} \mathrm{~B}_{3} \mathrm{H}_{5}\right)\right]_{16}(5)$ in $\mathrm{CDCl}_{3}$.

4 

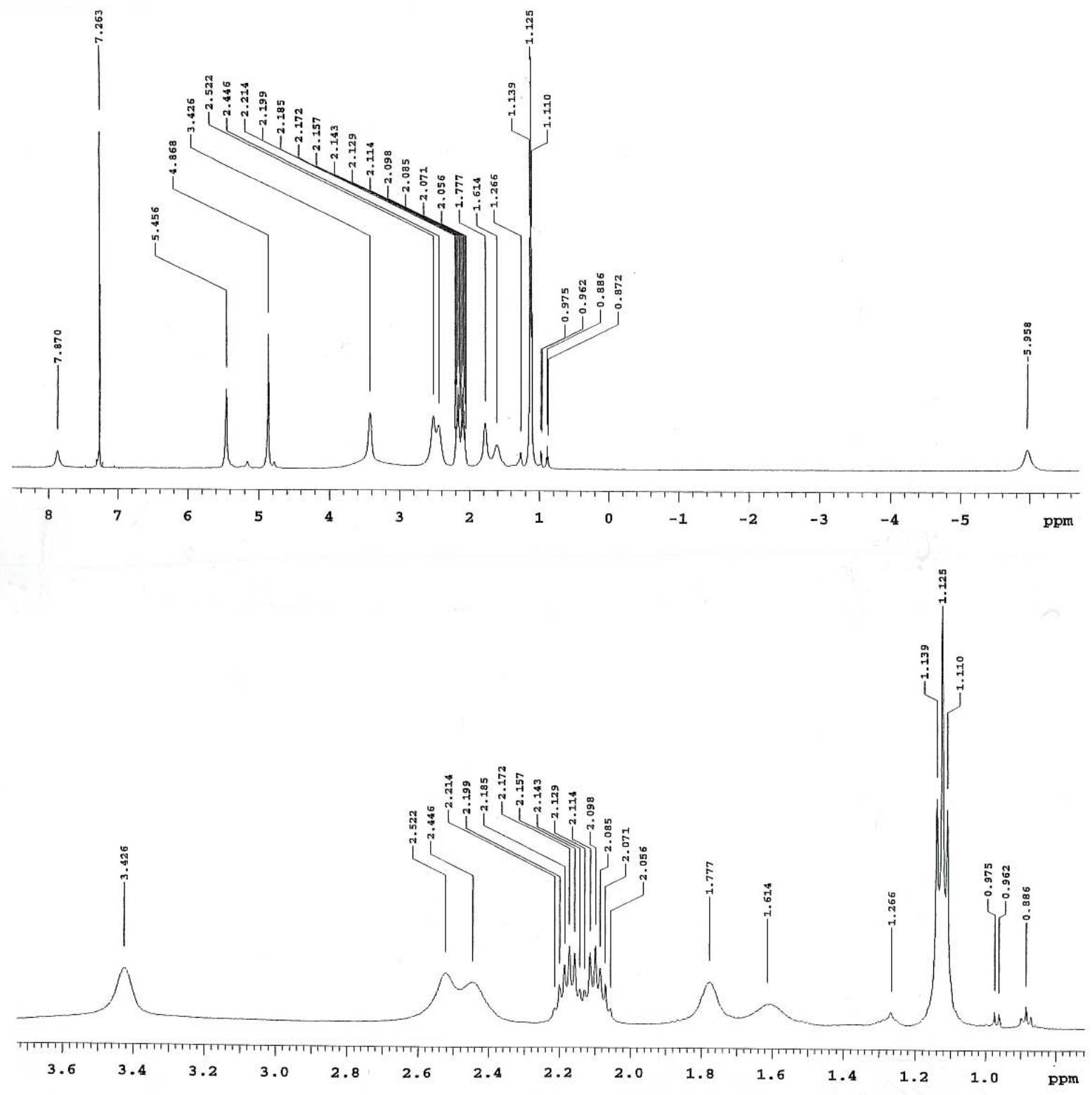

Figure 1(b). $500 \mathrm{MHz}{ }^{1} \mathrm{H}$ NMR spectra of DAB-dend-[NHCO-C $\left.\mathrm{H}_{4} \mathrm{Co}\left(2,3-\mathrm{Et}_{2} \mathrm{C}_{2} \mathrm{~B}_{3} \mathrm{H}_{5}\right)\right]_{32}(6)$ in $\mathrm{CDCl}_{3}$. 


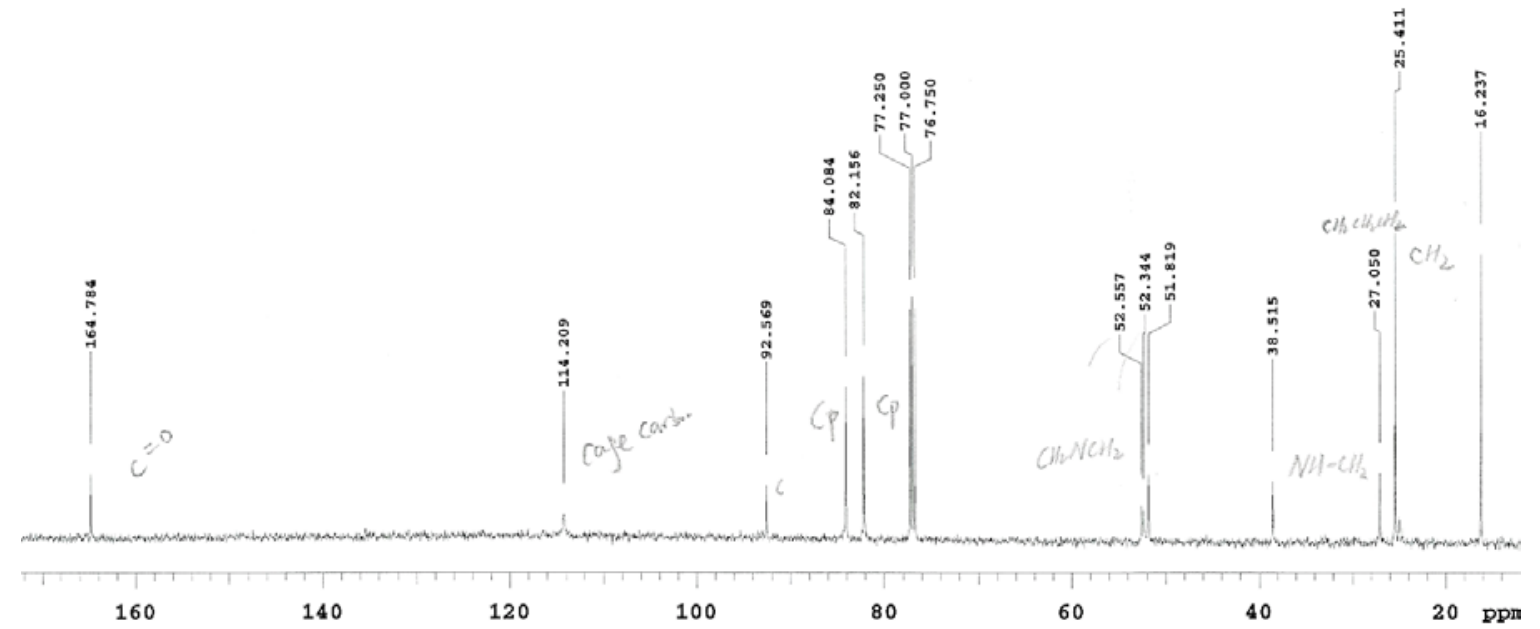

Figure 2(a). $125.8 \mathrm{MHz}{ }^{13} \mathrm{C}$ NMR spectra of dend-[NHC(O)- $\left.\mathrm{C}_{5} \mathrm{H}_{4} \mathrm{Co}\left(2,3-\mathrm{Et}_{2} \mathrm{C}_{2} \mathrm{~B}_{3} \mathrm{H}_{5}\right)\right]_{16}(5)$ in $\mathrm{CDCl}_{3}$.

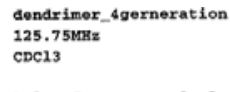

Pu1se Sequence: «2pul

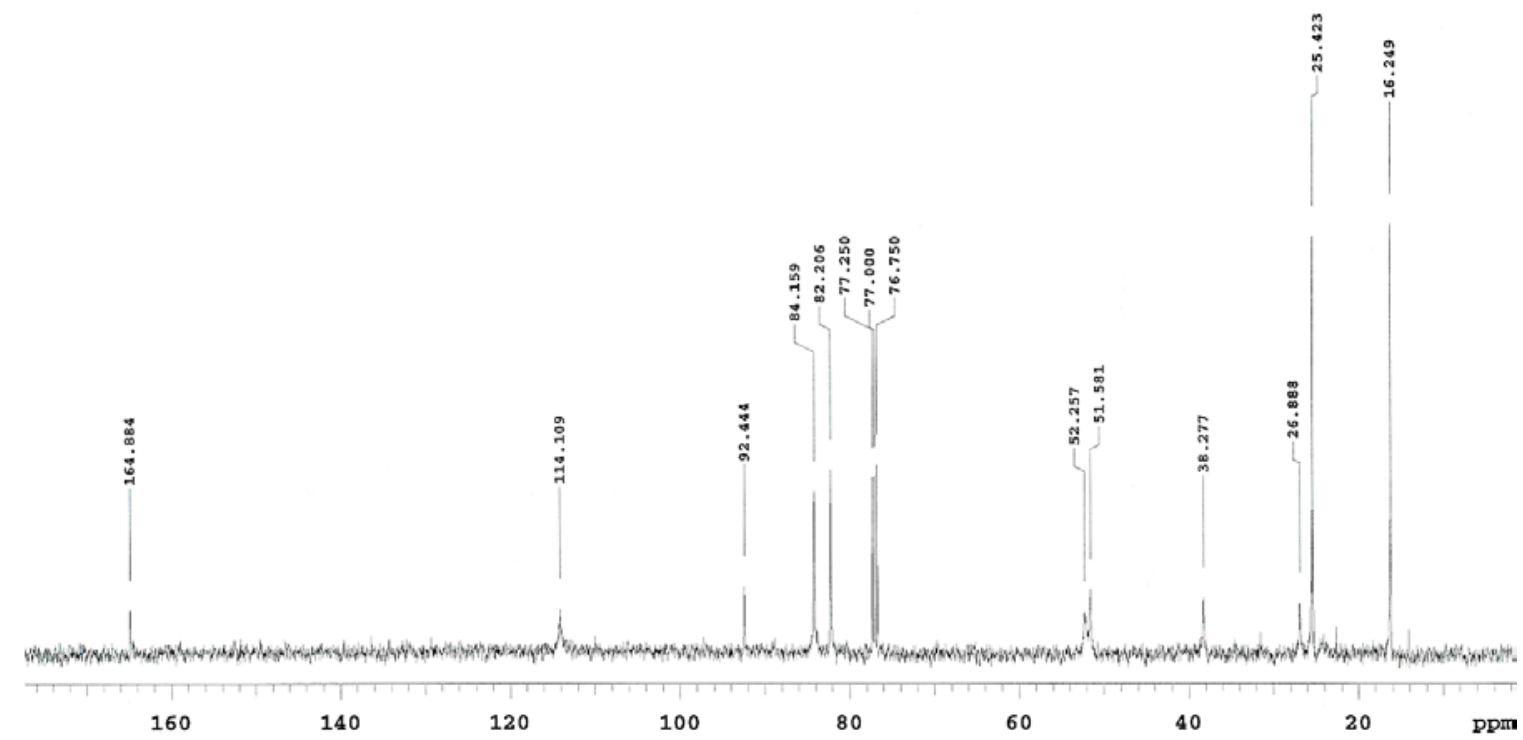

Figure 2(b). $125.8 \mathrm{MHz}{ }^{13} \mathrm{C}$ NMR spectra of DAB-dend-[NHCO-C $\left.{ }_{5} \mathrm{H}_{4} \mathrm{Co}\left(2,3-\mathrm{Et}_{2} \mathrm{C}_{2} \mathrm{~B}_{3} \mathrm{H}_{5}\right)\right]_{32}(\mathbf{6})$ in $\mathrm{CDCl}_{3}$. 
(a)

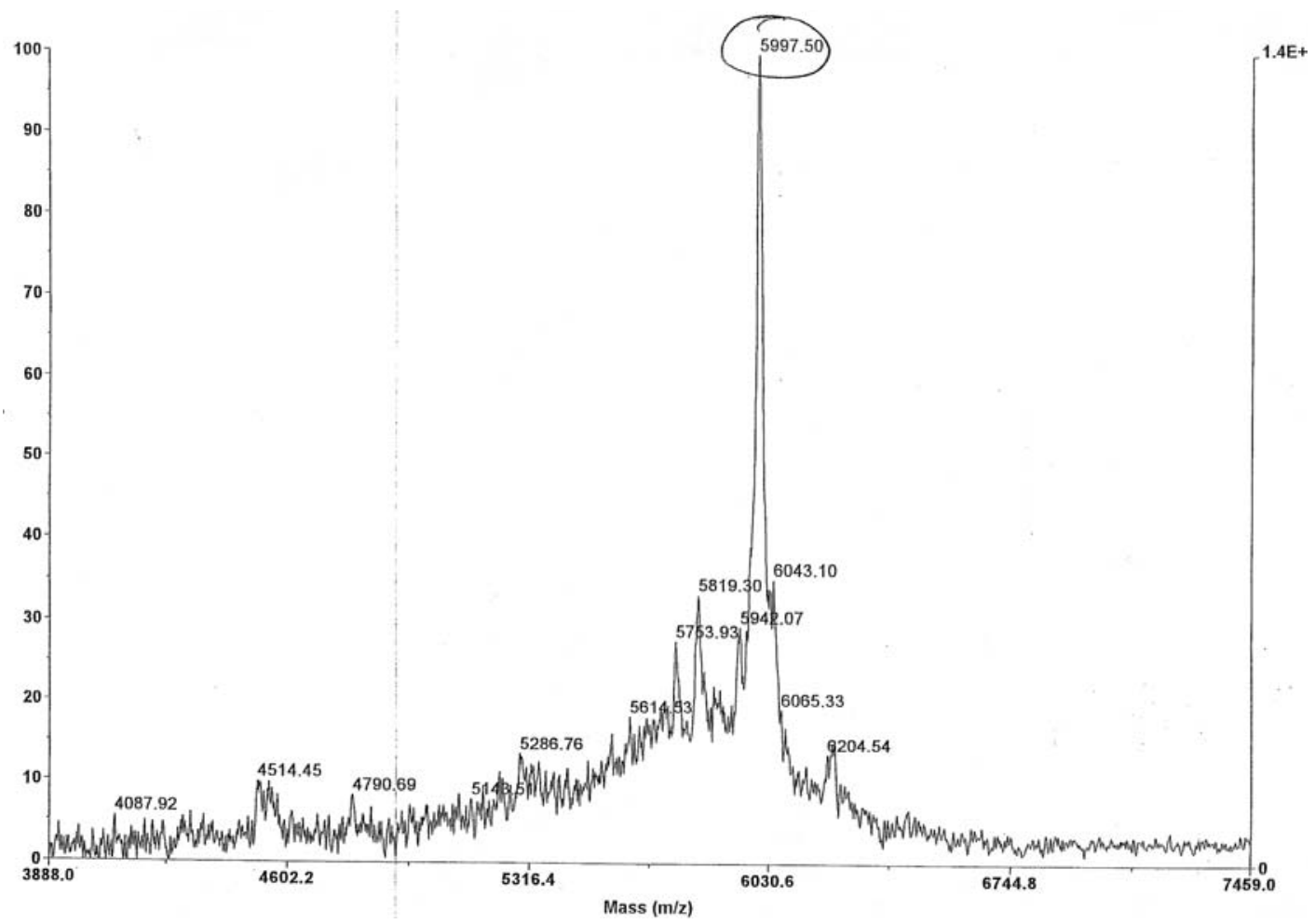

(b)

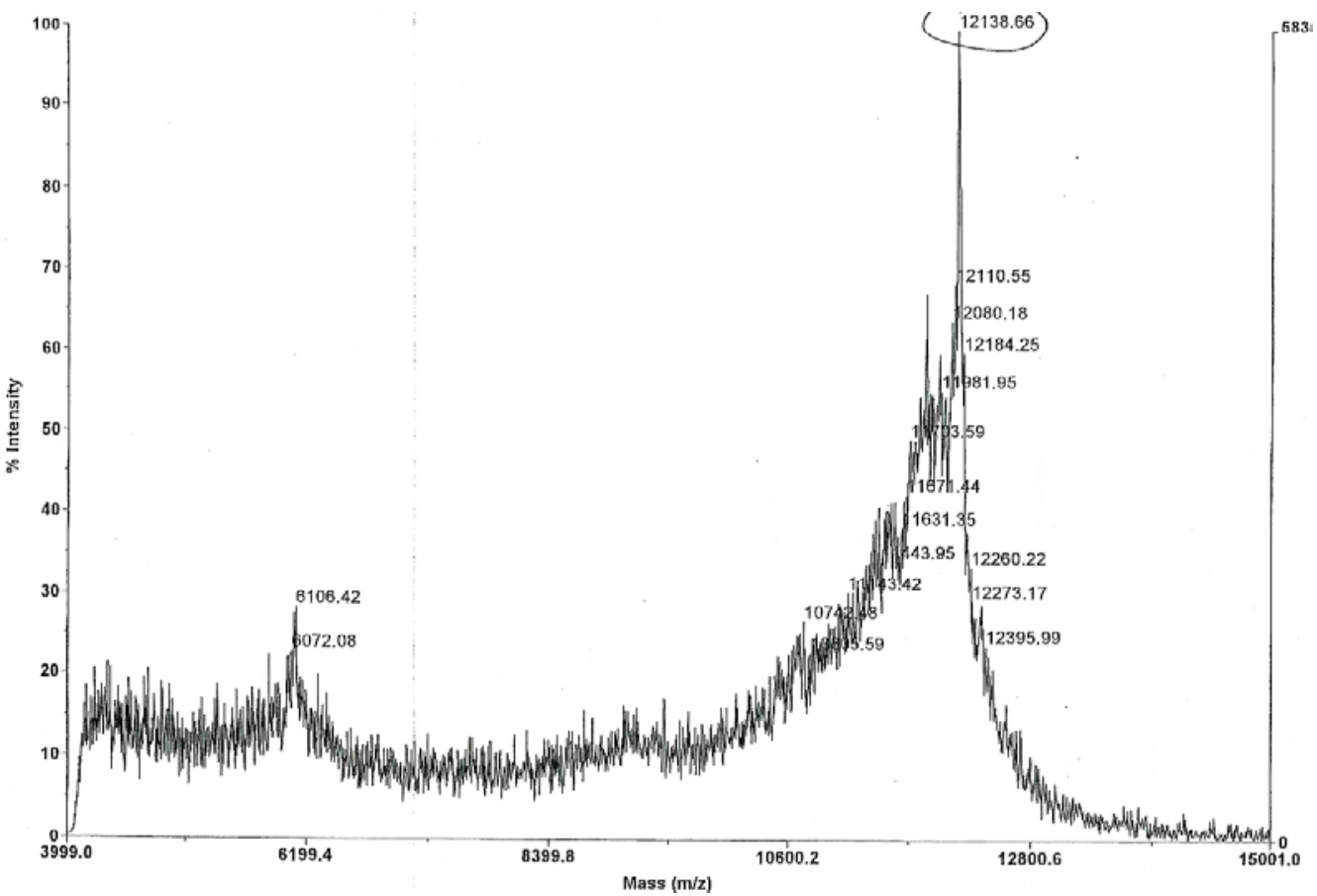

Figure 3. Matrix assisted laser desorption lonization-time of flight mass spectra of 5 (a) and 6 (b). 


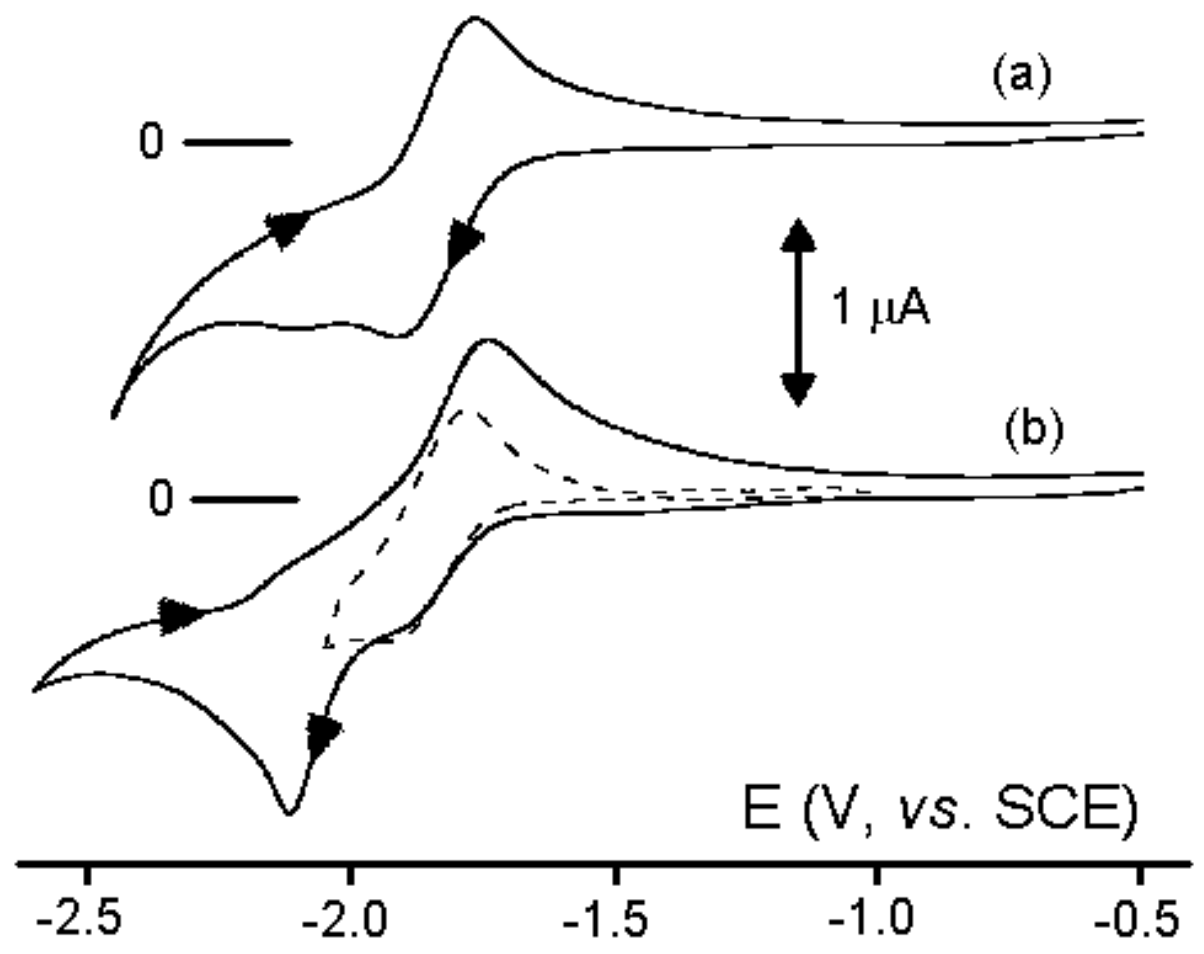

Figure 4. Cyclic voltammograms recorded at a gold millielectrode in THF solutions. (a) $5\left(6 \times 10^{-5} \mathrm{~mol} \mathrm{dm}^{-3}\right)$; (b) $6\left(3 \times 10^{-5} \mathrm{~mol} \mathrm{dm}^{-3}\right)$. [NBu $]\left[\mathrm{PF}_{6}\right]\left(0.2 \mathrm{~mol} \mathrm{dm}^{-3}\right)$ supporting electrolyte. Scan rate $0.05 \mathrm{Vs}^{-1}$.

1. James H. Davis, Jr., Ekk Sinn, and Russell N. Grimes, J. Am. Chem. Soc., 1989, 111, 4776.

2. Fabrizi de Biani, F.; Fontani, M.; Ruiz, E.; Zanello, P.; Russell, J. M.; Grimes R. N., Organometallics 2002, 21,, 4129 